\title{
Transarterial Radioembolization Versus Systemic Treatment for Hepatocellular Carcinoma with Macrovascular Invasion: Analysis of the U.S. National Cancer Database
}

\author{
Riad Salem and Ahmed Gabr \\ Department of Interventional Radiology, Northwestern University, Chicago, Illinois
}

$\mathbf{I}_{n}$ the December issue of The Journal of Nuclear Medicine, Ahn et al. (1) report the results of a National Cancer Database analysis comparing the trends and the outcomes of using transarterial radioembolization (TARE) and systemic therapy in the setting of locally advanced hepatocellular carcinoma (HCC) with portal vein thrombosis (PVT) in the presence of single or multiple tumors. The study shows not only the possible benefit of TARE in a real-life setting but also the trends and the factors associated with such treatment. The study spans an 8-y period (2010-2017) during which sorafenib was regarded as the gold standard systemic therapy for advanced HCC. Contextually, it should be highlighted that TARE has been Food and Drug Administration-approved since 1999, and its indication has been expanded to include locally advanced PVT since 2005. Hence, during the same time as this study, TARE has played a major role as the dominant arterial locoregional therapy in HCC with PVT, reflected in its increased use per Ahn's analysis.

This study, despite the limitations inherent in data from big national databases, showed 3 major key points. First, the increased adoption of TARE from $13 \%$ to $37 \%$ in advanced HCC is understandable, as the microembolic effect permits treatment in the presence of compromised portal flow. This is in direct contradistinction to chemoembolization, in which occlusion of the hepatic artery as part of treatment, in the presence of an occluded portal vein, risks inducing ischemic hepatitis. Also, the 40\%-50\% TARE response rate in this setting has far exceeded expectations, fueled increased use, and resulted in subsequent resection or liver transplantation, a novel observation resulting from the clinical introduction of TARE in the last decade. In fact, downstaging of patients with PVT to resection or transplantation has been demonstrated with TARE $(2,3)$. Of note, TARE has shown durable responses in the context of high response rates, similar to immunotherapies $(4,5)$. Second, the large sample size is a key factor that made it possible to capture the overall survival (OS) benefit of TARE over systemic therapy. In the recent phase III trials comparing TARE versus sorafenib (SARAH and SIRveNIB), OS differences were not captured, possibly because of insufficient sample sizes, postprogression therapy, treatment centers lacking the requisite expertise, and heterogeneous patient populations. Although a

\footnotetext{
Received Aug. 23, 2021; revision accepted Nov. 3, 2021.

For correspondence or reprints, contact Riad Salem (r-salem@ northwestern.edu).

COPYRIGHT (C) 2022 by the Society of Nuclear Medicine and Molecular Imaging
}

retrospective review of the National Cancer Database does overcome all of these issues, the large sample size can elicit differences in OS $(6,7)$. Third, the time frame in which the study was conducted suggests that most of the systemic therapy used was sorafenib. Therefore, the results can be extrapolated only to TARE versus sorafenib and not to TARE versus other systemic therapies.

The HCC community has witnessed the rapid approval of multiple systemic therapies, including regorafenib, lenvatinib, cabozantinib, ramucirumab, nivolumab, pembrolizumab, and ipilimumab, all of which provide more options for second- and third-line treatments (8). Furthermore, combinations such as atezolizumab and bevacizumab have been shown to be dramatically superior to sorafenib in terms of progression-free survival and OS (9). New trials have proven that not all systemic therapies for advanced HCC are the same. Novel systemic agents such as lenvatinib have been shown to be more effective at inducing tumor response than sorafenib as first-line treatment; other agents, such as regorafenib and cabozantinib, have been shown to be effective as second-line treatment at improving OS. Important recent observations have also been made with TARE, with the DOSISPHERE-01 trial showing that TARE more than doubled OS in a patient population with large tumors (mean, $10 \mathrm{~cm}$ ), two thirds of whom exhibited PVT, when personalized dosimetry was implemented (10).

Despite the thorough database analyses, there are several limitations. The lack of information about baseline tumor characteristics and burden and the extent of PVT, shown to significantly prognosticate outcomes in patients undergoing TARE, was a limitation (11). Another important limitation was the unavailability of data on subsequent second-line and third-line treatments, known to confound OS outcomes in clinical trials.

Although systemic therapies have significantly changed the landscape with level I evidence, there are still too many patients who do not respond and progress early. Advanced-HCC patients are often relegated only to systemic therapy; future investigations should focus on taking advantage of the durable antitumoral effect of local therapy in combination with systemic agents in advanced disease. Now with the ability to personalize dose and exceed 205 Gy with a durable effect, augmenting the effect of systemic therapy is not a remote possibility $(10,12)$. Well-designed trials are needed (13).

The overarching conclusion from this database analysis relates to the macroscale overview of patterns of clinical practice and national trends in TARE and systemic therapy use. If personalized decision making is our goal, we must significantly increase the 
baseline characteristics to be reported in all systemic therapy trials, similarly to what is done in locoregional therapies. Granularity of detail is needed, including index tumor size, number of lesions, overall hepatic burden, lobar or bilobar distribution, liver function, location of vascular invasion, and location of metastases. Combining extrahepatic disease and vascular invasion as a single category should be discouraged. Without this imaging detail as a form of biomarker, we will be unable to achieve the goal of individualizing patient treatment by baseline characteristics.

\section{DISCLOSURE}

Riad Salem is an advisor to Boston Scientific, Genentech, Eisai, Sirtex, Becton-Dickinson, Siemens, Cook, and AstraZeneca. No other potential conflict of interest relevant to this article was reported.

\section{REFERENCES}

1. Ahn JC, Lauzon M, Luu M, et al. Transarterial radioembolization versus systemic treatment for hepatocellular carcinoma with macrovascular invasion: analysis of the U.S. National Cancer Database. J Nucl Med. 2021;62:1692-1701.

2. Abouchaleh N, Gabr A, Ali R, et al. ${ }^{90} \mathrm{Y}$ radioembolization for locally advanced hepatocellular carcinoma with portal vein thrombosis: long-term outcomes in a 185-patient cohort. J Nucl Med. 2018;59:1042-1048.

3. Gabr A, Kulik L, Mouli S, et al. Liver transplantation following yttrium-90 radioembolization: 15-year experience in 207-patient cohort. Hepatology. 2021;73:998-1010
4. Gordon AC, Gabr A, Riaz A, et al. Radioembolization super survivors: extended survival in non-operative hepatocellular carcinoma. Cardiovasc Intervent Radiol. 2018;41:1557-1565.

5. Qaseem Y, Salem R. Observing durable responses and a prolonged survival tail in advanced hepatocellular carcinoma with portal vein invasion treated with Y90 radioembolization. Cardiovasc Intervent Radiol. 2020;43:1423-1424.

6. Vilgrain V, Pereira H, Assenat E, et al. Efficacy and safety of selective internal radiotherapy with yttrium-90 resin microspheres compared with sorafenib in locally advanced and inoperable hepatocellular carcinoma (SARAH): an openlabel randomised controlled phase 3 trial. Lancet Oncol. 2017;18:1624-1636.

7. Chow PKH, Gandhi M, Tan SB, et al. SIRveNIB: selective internal radiation therapy versus sorafenib in Asia-Pacific patients with hepatocellular carcinoma. J Clin Oncol. 2018;36:1913-1921.

8. Kudo M, Finn RS, Qin S, et al. Lenvatinib versus sorafenib in first-line treatment of patients with unresectable hepatocellular carcinoma: a randomised phase 3 noninferiority trial. Lancet. 2018;391:1163-1173.

9. Finn RS, Qin S, Ikeda M, et al. Atezolizumab plus bevacizumab in unresectable hepatocellular carcinoma. N Engl J Med. 2020;382:1894-1905.

10. Garin E, Tselikas L, Guiu B, et al. Personalised versus standard dosimetry approach of selective internal radiation therapy in patients with locally advanced hepatocellular carcinoma (DOSISPHERE-01): a randomised, multicentre, openlabel phase 2 trial. Lancet Gastroenterol Hepatol. 2021;6:17-29.

11. Spreafico C, Sposito C, Vaiani M, et al. Development of a prognostic score to predict response to yttrium-90 radioembolization for hepatocellular carcinoma with portal vein invasion. J Hepatol. 2018;68:724-732. 29331342

12. Salem R, Johnson GE, Kim E, et al. Yttrium-90 radioembolization for the treatment of solitary, unresectable HCC: the LEGACY study. Hepatology. 2021;74: 2342-2352.

13. Llovet JM, De Baere T, Kulik L, et al. Locoregional therapies in the era of molecular and immune treatments for hepatocellular carcinoma. Nat Rev Gastroenterol Hepatol. 2021;18:293-313. 